\title{
An Inexact Two-Stage Quadratic Program for Water Resources Planning
}

\author{
G. H. Huang ${ }^{1 *}$, Y. P. Li $^{1}$, H. N. Xiao ${ }^{2}$, and X. S. Qin ${ }^{1}$ \\ ${ }^{1}$ Faculty of Engineering, University of Regina, Regina, SK S4S 0A2, Canada \\ ${ }^{2}$ Department of Chemical Engineering, University of New Brunswick, 15 Dineen Drive, Fredericton, NB E3B 5A3, Canada
}

\begin{abstract}
An inexact two-stage stochastic quadratic programming (ITQP) model is developed for water resources management under uncertainty. The model is a hybrid of inexact quadratic programming and two-stage stochastic programming. It can deal with the uncertainties presented as both probabilities and intervals. Moreover, it can deal with nonlinearities in objective function to reflect the effects of marginal utility on the benefit and cost components. Using quadratic form in the objective function rather than linear one, the ITQP can minimize the unfair competition of water resources among multiple users under uncertain water conditions. In the modeling formulation, penalties are imposed when policies expressed as the promised water supply targets are violated. In its solution process, the ITQP model is transformed into two deterministic submodels based on an interactive algorithm and a derivative algorithm, which correspond to the lower and upper bounds of the desired objective. Interval solutions, which are feasible and stable in the given decision space, can then be obtained by solving the two submodels sequentially. The developed method is then applied to a case study of water resources management planning. The results indicate that reasonable solutions have been obtained. They can help provide bases for identifying desired water-allocation plans with maximized system benefit and minimized system-failure risk.
\end{abstract}

Keywords: decision making, inexact optimization, quadratic programming, two-stage stochastic, water resources

\section{Introduction}

Over the past decades, controversial and conflict-laden water allocation issues among competing municipal, industrial, and agricultural interests have raised increasing concerns (Huang and Chang, 2003). The growing population and shrinking water availability have exacerbated such competetions, particularly under varying natural conditions and deteriorating quality of water resources. On the other hand, uncertainties may exist in a number of the related system components, leading to complexities in generating desired decisions of water resources management; furthermore, such uncertainties may be multiplied by not only interactions among various sub-systems but also their associations with economic penalties if the promised targets are violated. Therefore, it is desired that effective approaches be developed to address these complexities and uncertainties.

Two-stage stochastic programming (TSP) method is effective for problems where an analysis of policy scenarios is desired and the right-hand-side coefficients are random with known probability distributions. In the TSP, a decision is first undertaken before values of random variables are known; then, after the random events have happened and their values are known, a second-stage decision can be made in order to minimize "penalties" that may appear due to any infeasibility (Loucks et al., 1981; Li et al., 2006). Previously, a number of researchers considered water resources management system uncertainties through applications of the TSP approaches

\footnotetext{
* Corresponding author: gordon.huang@uregina.ca
}

(Kovacs et al., 1986; Wang and Adams, 1986; Trezos and Yeh, 1987; Ruszczynski and Swietanowski, 1997; Ferrero et al., 1998; Huang and Loucks, 2000; Seifi and Hipel, 2001; Luo, et al., 2003; Maqsood et al., 2005; Li et al., 2006; Li and Huang, 2007). For example, Wang and Adams (1986) proposed a twostage optimization framework for the planning of optimal reservoir operations; Ferrero et al. (1998) examined hydrothermal scheduling of multi-reservoir systems using a twostage algorithm; Huang and Loucks (2000) proposed an inexact two-stage stochastic programming (ITSP) model for water resources management where the interval-parameter programming (IPP) method was introduced into the TSP framework. However, the ITSP method was based on an assumption that the objective function was linear, resulting in difficulties in dealing with such issues wherein marginal utility affect the revenue and/or cost coefficients in a TSP problem and thus make the relevant objective function nonlinear.

Quadratic program $(\mathrm{QP})$ is a useful tool for mathematical programming and has important application to systems analysis. It can reflect nonlinearity in the cost/benefit objectives, which exists in many environmental problems, and has global optimum under a number of system conditions (Hillier and Lieberman, 1986). For example, Rockafellar and Wets (1986) proposed a Lagrangian finite generation technique for solving linear-quadratic problems in two-stage stochastic programming; Shil'man (1992) used a stochastic quasigradient method for quadratic optimization under dependent observations; Huang et al. (1995) proposed an inexact quadratic program (IQP) through introduction of interval numbers into the QP framework. More recently, Chen and Huang (2001) extended 
the IQP work of Huang et al. (1995) and proposed a derivative algorithm method (DAM) for solving the IQP problem , which required much lower computational efforts than the previous algorithm and thus facilitated the IQP's application to large-scale problems. However, although the IQP approach could handle the interval uncertainties in the model's leftand/or right-hand sides as well as in the objective function, it had difficulties in dealing with uncertainties expressed as random variables and was lack of linkages to economic conesquences of violated policies.

Thus, as an extension of the previous works, this study aims to develop an inexact two-stage quadratic stochastic programming (ITQP) method for water resources planning under uncertainty. This is a hybrid in which approaches of inexact quadratic programming (IQP) and two-stage stochastic programming (TSP) will be integrated within a general optimization framework. The ITQP method can handle uncertainties expressed as both probability density functions and discrete intervals; moreover, it can deal with nonlinearities in objective function to reflect the impacts of marginal utility under stochastic conditions. A hypothetical case study will then be provided for demonstrating applicability of the developed method. The results will help water resources managers not only make decisions of water allocation but also identify desired water-allocation policies under maximized system benefit and minimized system-failure risk.

\section{Formulation of the ITQP Model}

Consider a problem wherein a water-resource manager is responsible for allocating water to multiple users under uncertainty. The total water supply available during the planning period has random features, and the users need to know how much water they can expect in order to make appropriate decisions to support their various activities and investments. Based on the local water resources management policy, a projected quantity of water can be allocated to each user. If this projected target is diverted, it will result in net benefits to the local economy; otherwise, penalties will be incurred. Moreover, in such a water resources management system, the impacts of marginal utility are desired to reflect since the benefit and penalty are expressed as inexact linear functions of water demand and shortage. This may lead to the relevant objective function nonlinearity (i.e. quadratic forms).

Consequently, the technique of interval-parameter quadratic programming (IQP) will be incorporated within a twostage stochastic programming (TSP) model to tackle the uncertainties expressed as interval and/or random and nonlinearities existing in the objective function. This may lead an inexact two-stage quadratic stochastic programming (ITQP) model. In the ITQP, a decision of water allocation target needs to be made before deterministic information about the future water flows is available, which is called the first-stage decision. Along with the time, when the actual water flows become available, a recourse action should then be taken; this is named the second-stage decision. The second-stage decision variables will be determined after the values of random vari- ables are available, so as to minimize penalties that may appear due to any infeasibility. Thus, we have:

$\operatorname{Max} f^{ \pm}=\sum_{i=1}^{m}\left(\alpha_{i}^{ \pm} T_{i}^{ \pm}+\beta_{i}^{ \pm}\right) T_{i}^{ \pm}-\sum_{i=1}^{m} \sum_{j=1}^{n} p_{j}\left(\sigma_{i t}^{ \pm} D_{i j}^{ \pm}+\delta_{i t}^{ \pm}\right) D_{i j}^{ \pm}$

subject to:

$q_{j}^{ \pm} \geq \sum_{i=1}^{m}\left(T_{i}^{ \pm}-D_{i j}^{ \pm}\right), \quad \forall j$,

$T_{i \max }^{ \pm} \geq T_{i}^{ \pm} \geq D_{i j}^{ \pm} \geq 0, \quad \forall i, j$.

where $f^{ \pm}=$expected net system benefit; $D_{i j}^{ \pm}=$amount by which water-allocation target $T_{i}^{ \pm}$is not met when the seasonal flow is $q_{j}^{ \pm}$with probability $p_{j}$ (decision variables); $i$ = water user, $i=1,2, \ldots, m ; j=$ the level of water availability, $j=1,2, \ldots, n ; p_{j}=$ the probability of occurrence for seasonal flow $q_{j}^{ \pm} ; q_{j}^{ \pm}=$random variable equal to total water available with probability $p_{j} ; T_{i}^{ \pm}=$fixed water-allocation target that is promised to user $i ; T_{i \max }^{ \pm}=$maximum allowable allocation amount for user i; $\alpha_{i t}^{ \pm}=$the slope of benefit curve for water demand for user $\mathrm{i}$ during period $\mathrm{t}\left(\alpha_{i t}^{ \pm<0}\right) ; \beta_{i t}^{ \pm}=$the intercept on the benefit axis for user i during period $\mathrm{t}\left(\beta_{i t}^{ \pm}>0\right)$; $\sigma_{i t}^{ \pm}=$the slope of penalty curve for water shortage for user i during period $\mathrm{t}\left(\sigma_{i t}^{ \pm}>0\right) ; \delta_{i t}^{ \pm}=$the intercept on the penalty axis for user i during period $\mathrm{t}\left(\delta_{i t}^{ \pm}>0\right)$. It is indicated that the relationship between benefit and demand is expressed as a monotonic decreasing inexact linear function, while the relationship between penalty and shortage is presented as a monotonic increasing one.

Symbols of $\alpha_{i t}^{ \pm}, \beta_{i t}^{ \pm}, \sigma_{i t}^{ \pm}, \sigma_{i t}^{ \pm}, T_{i}^{ \pm}, T_{i \max }^{ \pm}$and $D_{i j}^{ \pm}$denote interval parameters and variables, and superscripts ${ }^{-}$, and ' + ' represent lower and upper bounds of a parameter or variable. For example, letting $T_{i}^{-}$and $T_{i}^{+}$be lower and upper bounds of $T_{i}^{ \pm}$, respectively, we can have $T_{i}^{ \pm}=\left[T_{i}^{-}, T_{i}^{+}\right]$. Moreover, in model (1), water supply targets $T_{i}^{ \pm}$(i.e. waterallocation target that is promised to user $\mathrm{i}$ by the authority) need to be determined at the first-stage before the inflows are known, while water deficits $D_{i j}^{ \pm}$will be identified at the second-stage when the actual inflows are known. Thus, in this study, it is proposed that an optimized set of target values can be obtained by introducing $y_{i}$ as decision variables. According to Huang and Loucks (2000), let $T_{i}^{ \pm}$have a deterministic value of $T_{i}^{-}+\Delta T_{i} y_{i}$, where $\Delta T_{i}=T_{i}^{+}-T_{i}^{-}$and $y_{i} \in[0,1]$. Thus, model (1) can be converted to:

$$
\begin{aligned}
\operatorname{Max} & f^{ \pm}=\sum_{i=1}^{m}\left[\alpha_{i}^{ \pm}\left(T_{i}^{-}+\Delta T_{i} y_{i}\right)^{2}+\beta_{i}^{ \pm}\left(T_{i}^{-}+\Delta T_{i} y_{i}\right)\right] \\
& -\sum_{i=1}^{m} \sum_{j=1}^{n} p_{j}\left(\sigma_{i}^{ \pm} D_{i j}^{ \pm 2}+\delta_{i}^{ \pm} D_{i j}^{ \pm}\right)
\end{aligned}
$$

subject to:

$q_{j}^{ \pm} \geq \sum_{i=1}^{m}\left(T_{i}^{-}+\Delta T_{i} y_{i}-D_{i j}^{ \pm}\right), \quad \forall j$ 
$T_{i \max }^{ \pm} \geq T_{i}^{-}+\Delta T_{i} y_{i} \geq D_{i j}^{ \pm} \geq 0, \quad \forall i, j$

$0 \leq y_{i} \leq 1, \quad \forall i$

When $T_{i}^{ \pm}$approach their upper bounds (i.e. $y_{i}=1$ ), a high system benefit can be obtained if the water demands are satisfied, but a high penalty may have to be paid when the promised water is not delivered. Conversely, when $T_{i}^{ \pm}$reach their lower bounds (i.e. $y_{i}=0$ ), we may have a lower benefit but, at the same time, a lower risk of violating the promised targets (and thus lower penalty). This optimized set may correspond to the highest possible system benefit and reliability given the uncertain water-allocation targets.

When $T_{i}^{ \pm}$are known as deterministic values, model (2) can be transformed into two deterministic submodels which correspond to the upper and lower bounds of the desired objective-function value, respectively. The transformation process is based on the methods of interactive and derivative algorithms (Huang et al., 1995; Huang, 1996; Chen and Huang, 2001). In this study, since the penalty coefficients of $\sigma_{i}^{ \pm}$and $\delta_{i}^{ \pm}$that are related to the interval decision variables $\left(D_{i j}^{ \pm}\right)$have same sign (i.e. $\sigma_{i}^{ \pm}>0$ and $\delta_{i}^{ \pm}>0$ ), according to Chen and Huang (2001), all $D_{i j}^{-}$correspond to $f^{+}$, and all $D_{i j}^{+}$correspond to $f^{-}$. Consequently, interval solutions, which are feasible and stable in the given decision space associated with different system-failure risk levels, can then be obtained by solving the two submodels sequentially. Since the objective is to maximize net system benefit, submodel (1) corresponding to $f^{+}$(i.e. most desirable system objective) can be firstly formulated as follows:

$$
\begin{aligned}
\operatorname{Max} & f^{+}=\sum_{i=1}^{m}\left[\alpha_{i}^{-}\left(T_{i}^{-}+\Delta T_{i} y_{i}\right)^{2}+\beta_{i}^{+}\left(T_{i}^{-}+\Delta T_{i} y_{i}\right)\right] \\
& -\sum_{i=1}^{m} \sum_{j=1}^{n} p_{j}\left(\sigma_{i}^{-} D_{i j}^{-2}+\delta_{i}^{-} D_{i j}^{-}\right)
\end{aligned}
$$

subject to:

$$
\begin{aligned}
& q_{j}^{+} \geq \sum_{i=1}^{m}\left(T_{i}^{-}+\Delta T_{i} y_{i}-D_{i j}^{-}\right), \quad \forall j \\
& T_{i \max }^{+} \geq T_{i}^{-}+\Delta T_{i} y_{i} \geq D_{i j}^{-} \geq 0, \quad \forall i, j \\
& 0 \leq y_{i} \leq 1, \quad \forall i
\end{aligned}
$$

where $D_{i j}^{-}$and $y_{i}$ are decision variables. Let $D_{i j \mathrm{opt}}^{-}$and $y_{i \text { opt }}$ be solutions of submodel (1). The optimized water-allocation targets are $T_{i \mathrm{opt}}^{ \pm}=T_{i}^{-}+\Delta T_{i} y_{i \mathrm{opt}}$, which correspond to the extreme upper bound of system benefit under the uncertain inputs of water-allocation amounts. Then, we have the following submodel corresponding to $f^{-}$:

$$
\begin{aligned}
\operatorname{Max} & f^{-}=\sum_{i=1}^{m}\left[\alpha_{i}^{+}\left(T_{i}^{-}+\Delta T_{i} y_{i \mathrm{opt}}\right)^{2}+\beta_{i}^{-}\left(T_{i}^{-}+\Delta T_{i} y_{i \mathrm{opt}}\right)\right] \\
& -\sum_{i=1}^{m} \sum_{j=1}^{n} p_{j}\left(\sigma_{i}^{+} D_{i j}^{+2}+\delta_{i}^{+} D_{i j}^{+}\right)
\end{aligned}
$$

subject to:

$$
\begin{aligned}
& q_{j}^{-} \geq \sum_{i=1}^{m}\left(T_{i}^{-}+\Delta T_{i} y_{i \mathrm{opt}}-D_{i j}^{+}\right), \quad \forall j \\
& T_{i \max }^{-} \geq T_{i}^{-}+\Delta T_{i} y_{i \mathrm{opt}} \geq D_{i j}^{+} \geq 0, \quad \forall i, j \\
& D_{i j}^{+} \geq D_{i j \mathrm{opt}}^{-}, \quad \forall i, j
\end{aligned}
$$

where $D_{i j}^{+}$are decision variables. Let $D_{i j \mathrm{opt}}^{+}$be solutions of submodel (2). Thus, solutions for the ITQP-primal model under the optimized water allocation plan are:

$D_{i j \mathrm{opt}}^{ \pm}=\left[D_{i j \mathrm{opt}}^{-}, D_{i j \mathrm{opt}}^{+}\right], \quad \forall i, j$

$f_{\mathrm{opt}}^{ \pm}=\left[f_{\mathrm{opt}}^{-}, f_{\mathrm{opt}}^{+}\right]$

Then, the optimal water allocation plan and the expected net system benefit can be calculated as follows:

$A_{i j \mathrm{opt}}^{ \pm}=T_{i \mathrm{opt}}^{ \pm}-D_{i j \mathrm{opt}}^{ \pm}, \quad \forall i, j$

\section{Case Study}

Consider a case in which a water manager is responsible for allocating water in a dry season from an unregulated reservoir to three users: a municipality, an industrial concern, and an agricultural sector. All users want to know how much water they can expect over the planning period. If the promised water is delivered, a net benefit to the local economy will be generated for each unit of water allocated. However, if the promised water is not delivered, either the water must be obtained from higher priced alternatives or the demand must be curtailed by reduced production, resulting in a reduced net system benefit. Consequently, it is necessary for the available water to be effectively allocated to minimize any or all of the associated penalties or negative consequences. Tables 1 and 2 present the water resources available, target demands, and economic data.

The problem under consideration is how to effectively allocate water to the three users to achieve a maximum benefit under uncertainty, while incorporating water policies with the least risk of system disruption. Uncertainties exist in terms of intervals and probability distributions, and a link to a predefined policy is desired; moreover, nonlinear relationships may exist among many of the system components. Thus, the ITQP 
Table 1. Distribution of Stream Flows

\begin{tabular}{lll}
\hline Stream flow level & Probability $\left(p_{j}\right)$ & Stream flow, $q_{j}^{ \pm}\left(10^{6} \mathrm{~m}^{3}\right)$ \\
Low $(\mathrm{j}=1)$ & 0.1 & {$[3.0,6.5]$} \\
Low-medium $(\mathrm{j}=2)$ & 0.2 & {$[6.5,10.0]$} \\
Medium $(\mathrm{j}=3)$ & 0.4 & {$[10.0,14.0]$} \\
Medium-high $(\mathrm{j}=4)$ & 0.2 & {$[14.0,17.0]$} \\
High $(\mathrm{j}=5)$ & 0.1 & {$[17.0,20.0]$} \\
\hline
\end{tabular}

Table 2. Information of Water Allocation and Economic Implication

\begin{tabular}{|c|c|c|c|}
\hline \multirow[t]{2}{*}{ Activity } & \multicolumn{3}{|c|}{ Water users } \\
\hline & Municipal $(i=1)$ & Industrial $(\mathrm{i}=2)$ & Agricultural $(i=3)$ \\
\hline Maximum allowable allocation $\left(10^{6} \mathrm{~m}^{3}\right)$ & {$[5.0,7.0]$} & {$[7.0,10.0]$} & {$[12.0,15.0]$} \\
\hline Water allocation target $\left(10^{6} \mathrm{~m}^{3}\right)$ & {$[2.5,3.5]$} & {$[3.0,5.0]$} & {$[4.5,7.5]$} \\
\hline \multicolumn{4}{|c|}{ Net benefit when water demand is satisfied $\left(\$ / \mathrm{m}^{3}\right)$} \\
\hline$N B_{i}^{-} \quad$ (Lower-bound) & $-6.428 x+90$ & $-3.438 x+55$ & $-2.857 x+40$ \\
\hline$N B_{i}^{+} \quad$ (Upper-bound) & $-8.214 x+115$ & $-4.375 x+70$ & $-3.928 x+55$ \\
\hline \multicolumn{4}{|l|}{ Penalty when water is not diverted $\left(\$ / \mathrm{m}^{3}\right)$} \\
\hline$C_{i}^{-} \quad($ Lower-bound $)$ & $13.333 x+120$ & $5.385 x+70$ & $5.455 x+60$ \\
\hline$C_{i}^{+}$(Upper-bound) & $16.667 x+150$ & $6.923 x+90$ & $7.273 x+80$ \\
\hline
\end{tabular}

method is considered to be a feasible approach for this type of planning problem.

\section{Result and Discussion}

Table 3 presents the solutions obtained through the ITQP method. It is indicated that the solutions for the objective function value and most of the non-zero decision variables are intervals. Generally, solutions presented as intervals demonstrate that the related decisions should be sensitive to the uncertain modeling inputs. Deficits would occur if water resources available from the reservoir do not meet the user demands. In case of insufficient water, the allotment to the farmers should be first decreased but guaranteeing the minimum promised target. The municipal use should be first guaranteed since it brings the highest benefit when water demand is satisfied; meanwhile, it is subject to the highest penalty if the promised water is not delivered. In comparison, the industrial and agricultural uses correspond to the lower benefits and penalties. Figure 1 presents the water allocation targets and optimized water allocation patterns under different water-availability levels $\left(q_{j}^{ \pm}\right)$.

The results of the optimized water targets for the three users could be obtained through formula $T_{i \mathrm{opt}}^{ \pm}=T_{i}^{-}+\Delta T_{i} y_{i \mathrm{opt}}$. For example, the results of $y_{\text {lopt }}=y_{2 \text { opt }}=1.0$ indicate that the optimized water-allocation targets for the municipal and industrial users would equal $3.5 \times 10^{6}$ and $5.0 \times 10^{6} \mathrm{~m}^{3}$, corresponding to their upper-bound target values (i.e. $T_{1}^{+}$and $T_{2}^{+}$). This means that the manager is optimistic for water supply to the municipal and industrial users, and thus promises an upper-bound water quantity to the two users. Conversely, for agricultural sector, the result of $y_{3 \mathrm{opt}}=0$ indicates that the optimized water-allocation target reaches its lower-bound (i.e. $T_{3}^{-}=4.5 \times 10^{6} \mathrm{~m}^{3}$ ); this also implies that the manager has a conservative attitude toward water allocation to user 3 despite of water flow level. Each optimized water-allocation flow (listed in Table 3) is the difference between the fixed demand and the probabilistic shortage under a given stream condition and with an associated probability (i.e. $A_{i j \text { opt }}^{ \pm}=T_{i \text { opt }}^{-}+\Delta T_{i} y_{i \text { opt }}$ $-D_{i j \text { opt }}^{ \pm}$.

The solutions of $D_{i j}^{ \pm}$under the given targets reflect potential system condition variations caused by uncertain inputs. The lower-bound of $D_{i j}^{ \pm}$(i.e. $D_{i j}^{-}$) corresponds to a higher system benefit; in comparison, the upper-bound of $D_{i j}^{ \pm}$(i.e. $D_{i j}^{+}$) leads to a lower system benefit. For user 1 (i.e. municipal), the solution of $D_{11 \mathrm{opt}}^{ \pm}=[0.0,0.5] \times 10^{6} \mathrm{~m}^{3}$ means that, when seasonal flow level is low and associated with a probability of $10 \%$, there would be no shortage under advantageous conditions, and there would be a shortage of $0.5 \times 10^{6}$ $\mathrm{m}^{3}$ under demanding conditions. The results also indicate that there would be no shortage of water (in reference to the optimized water-allocation target of $3.5 \times 10^{6} \mathrm{~m}^{3}$ ) for the municipality under low-medium to high flows (i.e. $D_{12 \mathrm{opt}}^{ \pm}=D_{13 \mathrm{opt}}^{ \pm}$ $=D_{14 \mathrm{opt}}^{ \pm}=D_{15 \mathrm{opt}}^{ \pm}=0$ ). For user 2 (i.e. industrial), the solutions of $D_{21 \mathrm{opt}}^{ \pm}=[2.7,5.0] \times 10^{6}, D_{22 \mathrm{opt}}^{ \pm}=[1.0,3.0] \times 10^{6}$, and $D_{23 \mathrm{opt}}^{ \pm}=[0.0,1.2] \times 10^{6} \mathrm{~m}^{3}$ indicate that shortages may exist 
Table 3. Optimal Solutions Obtained from the ITQP Model

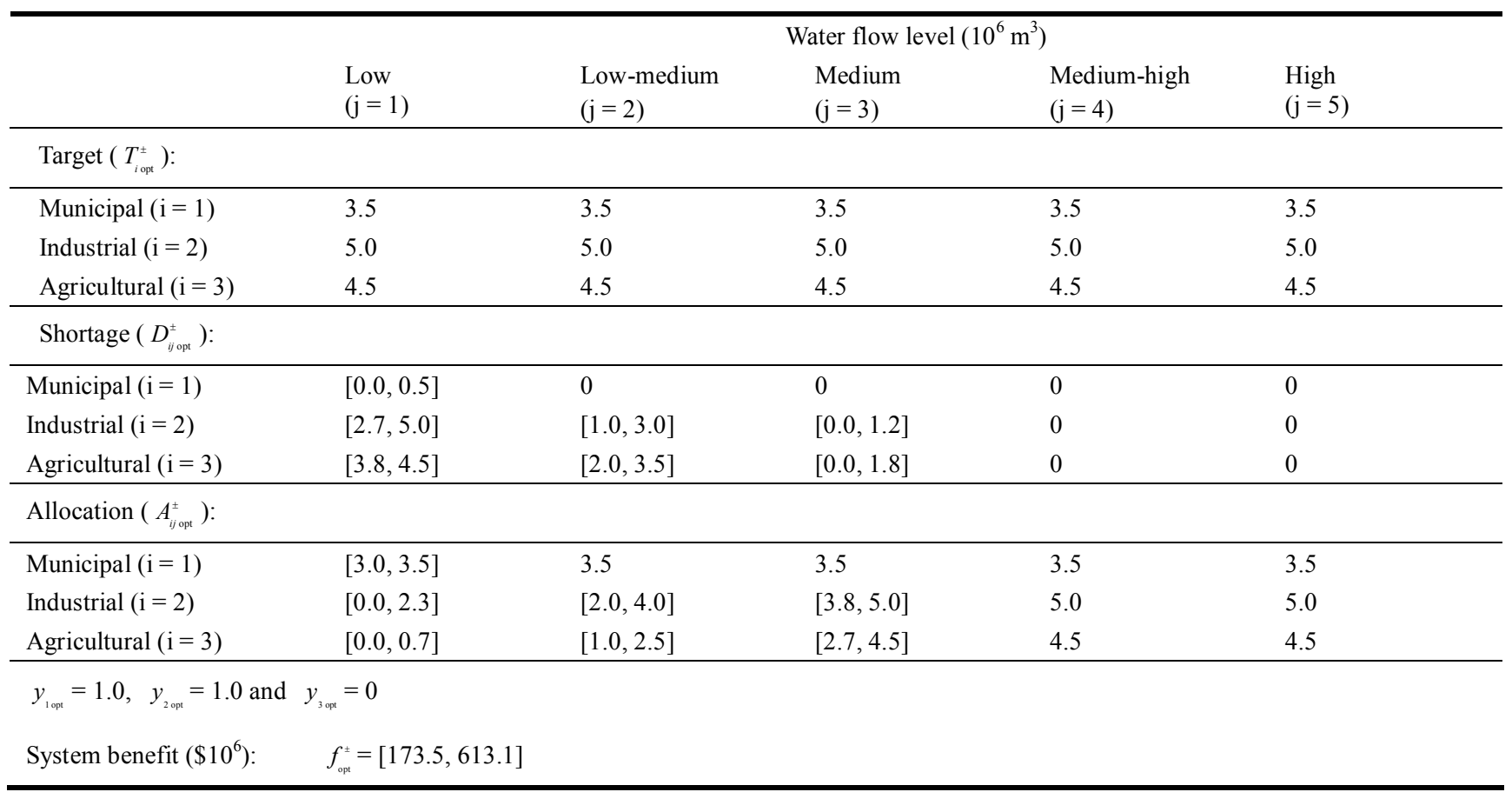

under low to medium flow levels, respectively; however, there would be no shortage under medium-high and high flow levels (i.e. $D_{24 \mathrm{opt}}^{ \pm}=D_{25 \mathrm{opt}}^{ \pm}=0$ ). For user 3 (i.e. agricultural), there would be no shortage under medium-high and high water-flow levels; however, the results indicate that, under low to medium flow levels, the shortages would be $[3.8,4.5]$ $\times 10^{6},[2.0,3.5] \times 10^{6}$ and $[0.0,1.8] \times 10^{6} \mathrm{~m}^{3}$, respectively.

The solution of $f_{\mathrm{opt}}^{ \pm}=\$[173.5,613.1] \times 10^{6}$ provides two extremes of the system benefit under the optimal water allocation pattern. As the actual value of each variable or parameter varies within its two bounds, the system benefit may change correspondingly between $f_{\text {opt }}^{-}$and $f_{\text {opt }}^{+}$with varied reliability levels. Planning for the lower-bound of the objective-function value will lead to a lower system benefit with a lower risk of violating the water-allocation target. Conversely, planning with a higher system benefit will correspond to a higher possibility of violating the water-allocation target when approaching the upper-bound of the objective-function value. Therefore, there is a tradeoff between the water-allocation benefit and the system-failure risk.

The problem was solved through the ITSP method (Huang and Locuks, 2000) without considering the effects of marginal utility on benefit and penalty. The main limitation of the ITSP is its over-simplification of the relationship between benefit and demand, as well as relationship between penalty and shortage. The ITSP method is based on an assumption that the effects of marginal utility are negligible and, thus, the relevant objective function is represented as linear form. However, the impacts of marginal utility may be significant when the limited water resources will be allocated among multiple competing users. This may make the ITSP method less realistic, leading to ineffectiveness in achieving the desired water-allocation schemes for decision-makers. In comparison, the ITQP method can effectively deal with such issues wherein marginal utility affect the benefit/cost coefficients in a mathematic programming problem. The results obtained through the ITQP method indicate that, when the deficit for the agricultural sector reaches a critical level, the allotment to the industry begins to decrease, resulting in some water allocation to the agricultural sector. It has demonstrated that, using quadratic form in the objective function rather than linear one, the ITQP can minimize the unfair competition of water resources among multiple users especially under water scarce conditions. It has advantages in better reflecting system benefit and/or cost variations and generating more reasonable and applicable solutions for decision makers. Thus, nonlinear problems provide more flexibility in the practical applications and allow decision-makers to take a more active role in controlling the system benefit and system-failure risk.

\section{Conclusions}

An inexact two-stage stochastic quadratic programming (ITQP) method has been developed and applied to water resources planning under uncertainty. This method improves upon the existing inexact two-stage linear programming approaches. It can deal with nonlinearities in the cost/benefit objective and uncertainties expressed as probability density functions and discrete intervals. Moreover, it can support the analysis of policy scenarios that are associated with economic penalties when the promised targets are violated. 


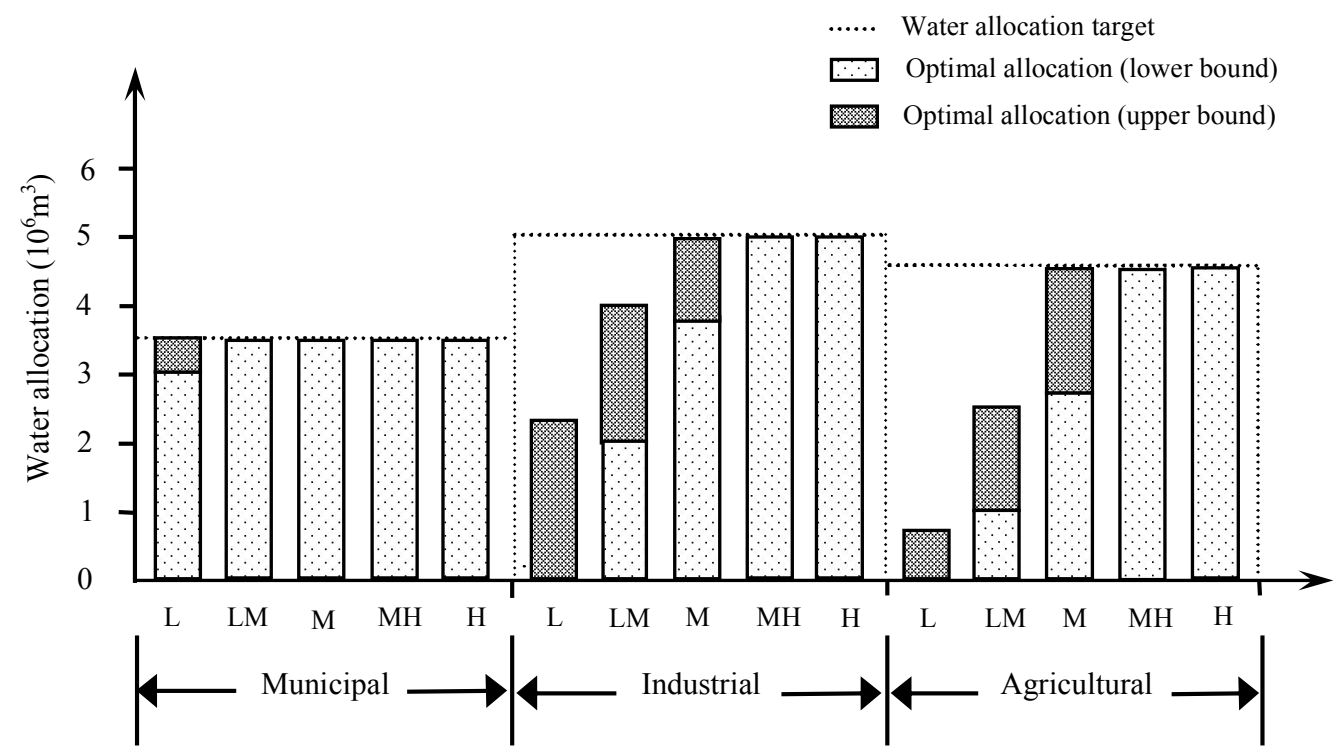

Figure 1. Optimized water allocation patterns under low (L), low-medium (LM), medium (M), medium-high (MH), and high (H) flows.

The ITQP method has been applied to a case study of water resources management. Based on an interactive algorithm and a derivative algorithm, the model can be transformed into two deterministic submodels which correspond to the upper and lower bounds of the desired objective-function value. Interval solutions, which are feasible and stable in the given decision space, can then be obtained by solving the two submodels sequentially. They can be used for generating decision alternatives and thus help waste managers to identify desired water-allocation plans with maximized system benefit and minimized system-failure risk.

In general, although this study is the first attempt for planning a water-resources management system through the developed ITQP approach, the results show that this hybrid is applicable and can be extended to other problems that involve complexities related to uncertainty, nonlinearity and policy analysis.

Acknowledgments. This research has been supported by the Major State Basic Research Development Program of China (2005CB724201 and 2005CB724207) and the Natural Science and Engineering Research Council of Canada.

\section{References}

Chen, M.J. and Huang, G.H. (2001). A derivative algorithm for inexact quadratic program-application to environmental decisionmaking under uncertainty, EJOR, 128, 570-586.

Ferrero, R.W., Rivera, J.F. and Shahidehpour, S.M. (1998). A dynamic programming two-stage algorithm for long-term hydrothermal scheduling of multireservoir systems, IEEE Transactions on Power Systems, 13, 1534-1540.

Hillier, F.S. and Lieberman, G.J. (1986) Introduction to Operations Research, fourth ed. Holden-Day, Oakland, CA.
Huang, G.H., Baetz, B.W. and Patry, G.G. (1995). Gery quadratic programming and its application to municipal waste management planning under uncertainty, Eng. Optimiz., 23, 210-223.

Huang, G.H. (1996). IPWM: an interval parameter water quality management model, Engineering Optimization, 26, 79-103.

Huang, G.H. and Loucks, D.P. (2000). An inexact two-stage stochastic programming model for water resources management under uncertainty, Civ. Eng. Environ. Syst., 17, 95-118.

Huang, G.H. and Chang, N.B. (2003). The perspectives of environmental informatics and systems analysis, JEI, 1, 1-6.

Kovacs, L., Boros, E. and Inotay, F. (1986). A two-stage approach for large-scale sewer systems design with application to the Lake Balaton resort area, EJOR, 23(2) 169-178.

Li, Y.P., Huang, G.H. and Nie, S.L. (2006). An interval-parameter multi-stage stochastic programming model for water resources management under uncertainty, Adv. Water Resour., 29, 776789.

Li, Y.P. and Huang, G.H. (2007). Interval-parameter two-stage stochastic nonlinear programming for water resources management under uncertainty, Water Resour. Manage., Available online.

Loucks, D. P., Stedinger, J.R. and Haith, D.A. (1981). Water Resource Systems Planning and Analysis, Prentice-Hall, Englewood Cliffs, N.J.

Luo, B., Maqsood, I., Yin, Y.Y., Huang, G.H. and Cohen, S.J. (2003). Adaption to climate change through water trading under uncertainty - an inexact two-stage nonlinear programming approach, JEI, 2(2), 58-68.

Maqsood, I., Huang, G.H. and Yeomans, J.S. (2005). An intervalparameter fuzzy two-stage stochastic program for water resources management under uncertainty, EJOR, 167, 208-225.

Rockafellar, R.T. and Wets, R.J.B. (1986). A Lagrangian finite generation technique for solving linear-quadratic problems in stochastic programming Math, Programming Study, 28, 63-93.

Ruszczynski, A. and Swietanowski, A. (1997). Accelerating the regularized decomposition method for two-stage stochastic linear problems, EJOR, 101, 328-342.

Seifi, A. and Hipel, K.W. (2001). Interior-point method for reservoir operation with stochastic inflows, J. Water Resour. Plann. 
Manage., 127(1), 48-57.

Shil'man, S.V. (1992). Stochastic quasigradient method for quadratic optimization under dependent observations, Automation and Remote Control, 53, 1881-1896.

Trezos, T. and Yeh, W.W.-G. (1987). Use of stochastic dynamic programming for reservoir management, Water Resour. Res., 23 ,
983-996.

Wang, D. and Adams, B.J. (1986). Optimization of real-time reservoir operations with Markov decision processes, Water Resour. Res., 22, 345-352.

Wang, L.Z., Fang, L. and Hipel, K.W. (2003). Water resources allocation: A cooperative game theoretic approach, JEI, 2, 11-22. 